Momčilo D. Sabié,

Beograd

\title{
IL TRAPASSATO REMOTO NELLE TRE REDAZIONI DEL ROMANZO MANZONIANO
}

Essendo il trapassato remoto un tempo che di secolo in secolo sta perdendo terreno (e lo può dimostrare anche l'impiego assai parco che se ne fa nelle opere letterarie moderne, per citare alcuni esempi: nel romanzo fogazzariano Daniele Cortis, ${ }^{1}$ pagg. 395, solo 17 volte, in La Ciociara ${ }^{2}$ di Alberto Moravia, pagg. 414, 18 volte, e nel romanzo di Ugo Pirro Mille tradimenti, ${ }^{3}$ pagg. 350 , cinque volte, in tutte queste opere esclusivamente nella temporale), crediamo utile analizzarlo nelle tre redazioni successive del romanzo manzoniano discutendone a una a una tutte le applicazioni, tanto piu che il Manzoni rappresenta uno scrittore che si proponeva di ravvicinare la lingua letteraria italiana alla parlata. ${ }^{4}$

Ritenendo che potesse risultare assai indicativo l'uso di dati statistici a conferma di certi risultati di carattere strettamente filologico, ci siamo decisi a seguire un metodo finora poco o per nulla praticato. Con ciò non intendiamo esaurito il problema; crediamo comunque che il largo uso di specchietti statistici di cui ci siamo valsi possa riuscire utile a una visione più chiara dell'intero problema e che i risultati numerici comportino conclusioni interessanti, se non decisive.

In base alle ricerche statistiche che abbiamo fatto nelle tre dette redazioni ${ }^{5}$ possiamo caratterizare l'uso del trapassato remoto cola seguente tabella (le percentuali seguono fra parentesi):

\begin{tabular}{cllllll}
\cline { 3 - 5 } redazione & $\begin{array}{l}\text { indipen- } \\
\text { denti }\end{array}$ & temporali & $\begin{array}{l}\text { conse- } \\
\text { cutive }\end{array}$ & $\begin{array}{l}\text { insieme } \\
\text { (temporali e } \\
\text { consecutive) }\end{array}$ & $\begin{array}{l}\text { totale } \\
\text { (indipen- } \\
\text { denti e di- } \\
\text { pendenti) }\end{array}$ \\
\hline a & $3(10,00)$ & $26(86,67)$ & $1(3,33)$ & $27(90,00)$ & $30(100)$ \\
b & 2 & $(5,26)$ & $36(94,74)$ & - & $36(94,74)$ & $38(100)$ \\
c & 2 & $(5,40)$ & $35(94,60)$ & - & $.35(94,60)$ & $37(100)$
\end{tabular}

1 Milano, 1896.

2 Milano, 1957.

3 Milano, 1959.

4 Cfr. Bruno Migliorini, Lingua e Cultura, Tumminelli, 1948, p. 31.

5 Nelle nostre ricerche ci siamo serviti delle tre redazioni del romanzo manzoniano in: Tutte le opere di Alessandro Manzoni, vol. II, tomo III - Fermo 
Pur permettendo le grammatiche italiane moderne questo tempo solamente nelle temporali, ${ }^{6}$ la nostra tabella ci mostra che il suo uso nelle principali, come lo troviamo p. e. nella letteratura antica italiana e nel Boccaccio, ${ }^{7}$ non era sparito. Intanto riesce chiaro che questo tempo sta sparendo, tanto più se sappiamo che nel Decamerone ${ }^{8}$ è stato adoperato in totale 168 volte, di cui nelle indipendenti 14 volte.

Tutti questi dati possono aiutarci a capire la natura di questo tempo e la ragione della sua lenta ma permanente sparizione. Perciò, prima di tentare di venire a certe conclusioni, ci proponiamo a discutere il suo uso nel romanzo manzoniano: I. nelle dipendenti; II. nelle indipendenti.

Quanto alle dipendenti, dobbiamo limitarci esclusivamente alle temporali, essendovi il trapassato remoto adoperato una sola volta fuori di questo, cioè nella consecutiva della prima redazione: »Allora Fermo tor nava in sé, e si sforzaoa di perdonare: di modo che, in quel viaggio, egli ebbe ammazzato in cuore Don Rodrigo e risuscitatolo almeno venti volte." - (a, III, V, p. 413). Ma l'applicazione del tempo in parola in una dipendente di questo tipo si spiega colla sua vicinanza alle temporali.

L'uso del trapassato remoto nelle temporali del romanzo manzoniano lo possiamo raffigurare secondo il tipo della frase, cioè secondo la congiunzione, con la seguente tabella:

il tipo

\begin{tabular}{|c|c|c|c|c|c|c|c|c|c|c|c|c|c|}
\hline \multirow[b]{2}{*}{ redazione } & \multicolumn{4}{|c|}{ «giunto che fu» } & \multicolumn{3}{|c|}{ quando } & \multicolumn{3}{|c|}{ appena } & \multicolumn{3}{|c|}{ poi che } \\
\hline & $\begin{array}{l}\mathscr{U} \\
\mathbb{D} \\
\mathbb{D} \\
\stackrel{D}{0}\end{array}$ & 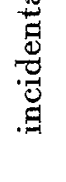 & $\begin{array}{l}\stackrel{0}{0} \\
\stackrel{5}{50} \\
\stackrel{6}{0}\end{array}$ & 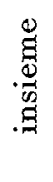 & 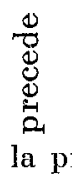 & 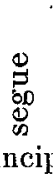 & 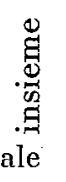 & 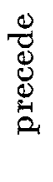 & $\begin{array}{l}\mathbb{S}_{80} \\
\stackrel{0}{\mathscr{D}}\end{array}$ & 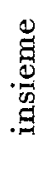 & $\begin{array}{l}\stackrel{0}{0} \\
\mathbb{d} \\
\mathbb{d} \\
\stackrel{0}{0}\end{array}$ & 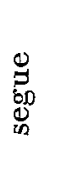 & 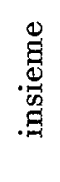 \\
\hline $\mathrm{a}$ & 2 & 2 & 一 & 4 & 13 & 2 & 15 & 4 & - & 4 & 1 & - & 1 \\
\hline b & 8 & 3 & 1 & 12 & 11 & 2 & 13 & 6 & - & 6 & 1 & - & 1 \\
\hline c & 6 & 1 & - & $?$ & 14 & 1 & 15 & 7 & - & 7 & - & - & - \\
\hline
\end{tabular}

e Lucia (1821-1823) - segnato a; tomo II - I Promessi Sposi (1825-1827) segnato $b$; tomo I - I Promessi sposi (1840) - segnato $c$; Milano, Mondadori, 1959.

- Cfr. S. Battaglia - V. Pernicone, La grammatica italiana, seconda edizione, Torino, 1955, p. 372-73.

${ }_{7}$ Cfr. Raffaello Fornaciari, Sintassi italiana dell'uso moderno, seconda edizione, Firenze, 1879, p. 180-81.

${ }^{8} \mathrm{Ci}$ siamo serviti del Decamerone nell'edizione: Giovanni Boccaccio, Decameron, Filocolo, Ameto, Fiammetta, Milano-Napoli, Riccardo Ricciardi, 1952. - Cfr. M. D. Savić, Jedno vreme u»Dekameronu« - prilog sintaksi trapasata remota ['Un tempo nel «Decamerone» - contributo alla sintassi del trapassato remoto'], Živi jezici IV, 1-4, 1962, str. 25-35. 


\begin{tabular}{|c|c|c|c|c|c|c|c|c|c|c|c|c|c|}
\hline \multirow[b]{2}{*}{$\begin{array}{l}\text { leda- } \\
\text { zione }\end{array}$} & \multicolumn{3}{|c|}{ dopo che } & \multicolumn{3}{|c|}{ tosto che } & \multicolumn{3}{|c|}{$\begin{array}{l}\text { fino che } \\
\text { (finché) }\end{array}$} & \multicolumn{3}{|c|}{ tanto che } & \multirow[b]{2}{*}{$\frac{0}{\frac{\pi}{\pi}}$} \\
\hline & 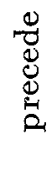 & 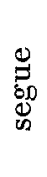 & 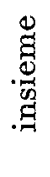 & 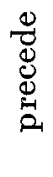 & 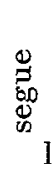 & $\begin{array}{c}\stackrel{9}{g} \\
. \mathbb{1} \\
. 尹 \\
\text { prin }\end{array}$ & 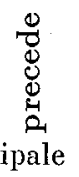 & 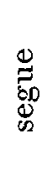 & 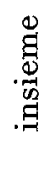 & 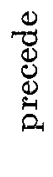 & 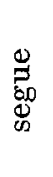 & 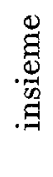 & \\
\hline a & 1 & -- & 1 & 1 & - & 1 & - & - & - & $\ldots$ & - & -- & 26 \\
\hline b & 1 & 1 & 2 & - & - & - & - & 1 & 1 & $\ldots$ & 1 & 1 & 36 \\
\hline $\mathrm{c}$ & 2 & 1 & 3 & 一 & - & - & - & 2 & 2 & - & 1 & 1 & 35 \\
\hline
\end{tabular}

Sebbene possa sembrare che una discussione di questa sorta sia inutile, noi la troviamo molto importante, perché ci dà un'idea abbastanza chiara del valore sintattico del trapassato remoto. Come vediamo, nella maggior parte dei casi trovati la temporale precede la principale. Questo momento ci riuscirà più evidente se ripetiamo la stessa tabella in $\%$ :

il tipo «giunto che fu» quando appena poi che

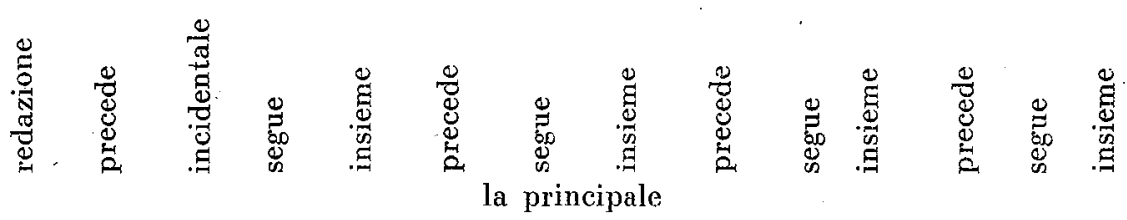

\begin{tabular}{lrllllllllllll}
\hline $\mathrm{a}$ & 7,69 & 7,69 & - & 15,38 & 50,00 & 7,69 & 57,69 & 15,38 & - & 15,38 & 3,85 & - & 3,85 \\
$\mathrm{~b}$ & 22,22 & 8,33 & 2,78 & 33,33 & 30,54 & 5,56 & 36,10 & 16,67 & - & 16,67 & 2,78 & - & 2,78 \\
$\mathrm{c}$ & $\mathbf{1 7 , 1 4}$ & 2,86 & - & 20,00 & 40,00 & 2,86 & 42,86 & 20,00 & - & 20,00 & - & - & -
\end{tabular}

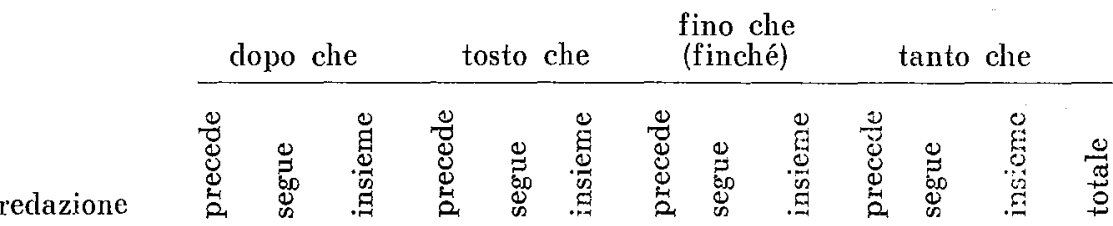
la principale

$\begin{array}{llllllllllllll}\mathrm{a} & 3,85 & - & 3,85 & 3,85 & - & 3,85 & - & - & - & - & - & 100 \\ \mathrm{~b} & 2,78 & 2,78 & 5,56 & - & - & - & - & 2,78 & 2,78 & \mathbf{2} & 2,78 & 2,78 & 100 \\ \mathrm{c} & 5,71 & 2,86 & 8,57 & - & - & - & - & 5,71 & 5,71 & - & 2,86 & 2,86 & 100\end{array}$

Forse non sarà senza importanza vedere su una tabella, non tenendo più conto delle congiunzioni e dei tipi della temporale, quante volte in totale la temporale preceda la principale, cioè quante volte vi sia incisa ${ }^{9}$

${ }^{9}$ Coi termini kincidentale», «incisa», «inserita» pensiamo ai casi come: «Don Abbondio finito ch'ebbe di scrivere rilesse lentamente, (a, I, VII, p. 126). 
e quante volte la segua. Questa tabella potrebbe esser data così (la percentuale segue fra parentesi):

la temporale

\begin{tabular}{cllll}
\cline { 2 - 4 } redazione & $\begin{array}{l}\text { precede la } \\
\text { principale }\end{array}$ & $\begin{array}{l}\text { è inserita nel- } \\
\text { la principale }\end{array}$ & $\begin{array}{l}\text { segue la } \\
\text { principale }\end{array}$ & totale \\
\hline a & $22(84,62)$ & $2(7,69)$ & $2(7,69)$ & $26(100)$ \\
b & $27(75,00)$ & $3(8,33)$ & $6(16,67)$ & $36(100)$ \\
c & $29(82,85)$ & $1(2,86)$ & $5(14,29)$ & $35(100)$
\end{tabular}

Analizzando la situazione esposta in questa tabella veniamo a conoscenza del fatto che le proposizioni in cui la temporale viene dopo l'indipendente (in totale 13 volte nelle tre redazioni) sono senza maggiore portata. E appena dopo questa constatazione possiamo farci una domanda sulla natura del trapassato remoto: si tratta forse di un tempo relativo, applicato cioè nella maggioranza dei casi come preterito del passato, ovvero di un tempo assoluto a cui questa applicazione viene attribuita dalla posizione in cui si trova di solito?

Noi siamo propensi a vedere in questa forma verbale un tempo che in sé stesso porta il suo significato assoluto e che ha assunto il valore relativo grazie alla posizione in cui sta prevalentemente. Questa affermazione richiede però un'ulteriore discussione, specialmente per le indipendenti, discussione alla quale torneremo fra breve. Qui invece aggiungiamo, per render più chiara la nostra analisi, che il trapassato remoto si può trovare di rado nella temporale in correlazione con un tempo della principale, che non sia il passato remoto. Ce lo indica la tabella seguente:

\begin{tabular}{cllll} 
redazione & $\begin{array}{l}\text { passato } \\
\text { remoto }\end{array}$ & $\begin{array}{l}\text { trapassato } \\
\text { remoto }\end{array}$ & imperfetto & $\begin{array}{l}\text { principale } \\
\text { senza verbo } \\
\text { finito }\end{array}$ \\
\hline a & $25(96,43)$ & $1(3,57)$ & - & - \\
b & $34(94,44)$ & - & $1(2,78)$ & $1(2,78)$ \\
c & $32(91,42)$ & - & $1(2,86)$ & $2(5,72)$
\end{tabular}

Possiamo farci ora un'altra domanda: non si tratta forse solo di una forma alterata del passato remoto, cioè di uno spostamento dei tempi che ha avuto luogo nel periodo storico della lingua italiana? Noi siamo portati ad affermarlo, e ciò sarà più credibile se diamo un'altra tabella da cui riesce evidente che nella maggior parte dei casi in cui viene adoperato il passato remoto la principale e la dipendente sono legate con lo stesso soggetto: 
redazione

soggetto

soggetti

il soggetto della principale fa

comune

differenti

parte di quello

$\begin{array}{llll}\mathrm{a} & 13(46,43) & 13(46,43) & 2(7,14) \\ \mathrm{b} & 22(61,11) & 10(27,78) & 4(11,11) \\ \mathrm{c} & 21(60,00) & 11(31,43) & 3(8,75)\end{array}$

Se nella prima redazione le temporali con soggetto diverso ammontano quasi al $50 \%$, nelle due redazioni successive troviamo una situazione ben diversa, cioè prevalgono notevolmente $\mathrm{i}$ casi in cui la temporale e l'indipendente sono legate con oggetto comune.

Comunque, non saremo in grado di far maggior luce su questo tempo se non esaminiamo la sua applicazione nelle indipendenti.

\section{II}

$\mathrm{Ci}$ parrà forse a prima vista che nelle tre redazioni del romanzo manzoniano non ci sia nessuna differenza riguardo all'uso del trapassato remoto nelle principali. La seconda delle tabelle incluse c'insegna invece che nella prima redazione questo numero non è senza importanza, ammontando al $10 \%$. Nelle due redazioni posteriori il quandro cambia essenzialmente, essendo il numero totale dei trapasati remoti aumentato e quello delle indipendenti diminuito. Questo, però, ci dà soltanto l'aspetto esterno del fenomeno. Il quadro strutturale delle proposizioni in cui appare il trapassato remoto è molto più significativo,

亡̀ ben conosciuto che il Manzoni rifece la prima redazione radicalmente, mentre, preparando l'ultima, diede alla seconda solo qualche piccolo ritocco. Tuttavia, quanto all'uso del trapassato remoto, le tre redazioni sono abbastanza differenti.

Nella prima redazione le indipendenti col trapassato remoto possono esser divise in due tipi:

1. E non l'ebbe appena proferita, che sentendo cessato il pericolo imminente, e vedendo che Fermo non aveva più pretesto di minacciarlo, la paura si cangiò in collera e cominciò a rimproverarlo. (a, I, II, p. 38); Ebbe appena don Abbondio proferite queste ultime parole che se ne penti, (a, III, IV, p. 389).

Come vediamo, in ambedue i casi non si tratta dell'uso enfatico del trapassato remoto. Pur essendo nella principale, esso indica l'azione che precede quella della dipendente. Si può aver l'impressione che in questi

10 Qui pensiamo ai casi come: «Tornati che furono al palazzo, il Griso rese conto, (c, VII, p.115), dove «il Griso» (soggetto della principale) fa parte di quello della temporale. 
tipi di frase si tratti solo di uno scambio dei posti: la principale viene invece della dipendente e viceversa, cioè: «Appena ebbe proferite queste ultime parole, don Abbondio se ne pentì.» Questa spiegazione però potrebbe esser accettata soltanto dal punto di vista logico, avendo avuto il trapassato remoto una ricca tradizione di questa specie nella letteratura antica taliana, p.e. nel Decamerone: $\gg N e$ ebbe guari cavato, che ella tropò il corpo del suo misero amante...»(IV, 5, p. 317). Un tipo contrario, che non si trova nel romanzo manzaniano, e in cui incontriamo davvero uno scambio dei posti, sarebbe, p. e., quello del Decamerone: «... né prima peduta l'ebbe, che egli fieramente assalito fu dalla concupiscenza carnale.« ( $\mathrm{I}, 2, \mathrm{p} .47)$.

2. Quando questi ebbe terminato, Fermo ebbe inteso: (a, I, III, p. 51).

Questo tipo d'applicazione del trapassato remoto si avvicina all'ultimo tipo boccaccesco, pur non essendogli assolutamente analogo. L'ultimo esempio ci mostra la possibilità di adoperare questo tempo nella indipendente con un significato speciale, cioè come un passato remoto pronunciato un po' più espressivamente con lo scopo di sottolineare meglio l'azione finita in un dato momento, il quale momento non dovrebbe esser sempre posto nel passato, ma anche nel presente ovvero nel futuro. Ma tutte queste affermazioni richiedono un'ulteriore discussione sul significato essenziale del passato remoto; e su questo punto dobbiamo gettar un po' di luce.

Nel rifacimento della prima redazione del suo romanzo il Manzoni ha omesso tutti gli episodi in cui si trovano le indipendenti citate col trapassato remoto. Eppure la seconda redazione cindica che egli vedeva nel trapassato remoto anche un tempo assoluto. Anche in questa rielaborazione incontriamo due tipi di trapassato remoto:

1. Intanto il vicario delle monache ebbe rilasciata l'attestazione necessaria, e venne la licenza di tenere il capitolo per l'accettazione di Gertrude. (b, X, p. 179).

L'applicazione del trapassato remoto in questa principale è molto significativa. Se nell'ultimo esempio citato della prima redazione potevamo vedere nel trapassato remoto ancora un preterito del passato trasferitosi nella indipendente (forse simile al trapassato prossimo), la proposizione sopraccitata ci dà un'altra idea, tanto più che questa volta il trapassato remoto si trova allo stesso punto temporale (Zeitstufe) col passato remoto, cioè la prima forma verbale non presenta nessun preterito riguardo alla seconda. A nostro parere, nella prima forma si tratterebbe soltanto di un passato remoto più espressivo. E questa espressività non si riattacca solo alla forma che appare sotto la veste del trapassato remoto, ma dà lo stesso colore anche al passato remoto che le viene dopo. Questa costruzione, d'origine stilistica, che si è acquistata anche il valore sintattico col Boccaccio o forse prima di lui, ci sarà più chiara esprimendola con la formula: $q(a+b)$, dove in $q$ vediamo l'ele- 
mento d'enfasi, cioè l'ausiliare $e b b e$, che non si riferisce solo al participio che precede (rilasciata - a), ma anche al passato remoto (venne - b) che gli succede. Quest'idea ci viene suggerita dall'impossibilità di trovare due trapassati remoti in una indipendente. ${ }^{11}$

Che differenza allora esiste tra il trapassato remoto in questa applicazione e in quella delle temporali? Nessuna. Noi non siamo d'accordo con quelli che vi vedono due applicazioni diverse di questa forma. ${ }^{12}$ Ripetiamo: esiste un'unica applicazione del trapassato remoto, il quale non rappresenta mai il preterito del passato; se lo troviamo di solito in quest'ultima applicazione, ciò deriva esclusivamente dalla sua posizione e non dalla sua natura temporale.

Come abbiamo detto, in questo fenomeno noi troviamo nell'origine un'applicazione puramente stilistica, che aveva in prima linea un certo valore soggettivo. Grazie all'uso quotidiano, esso si andò modificando a poco a poco finché non acquistò un significato sintattico, tanto più che era adoperato attraverso molte generazioni. Questo processo doveva esser terminato già prima del Boccaccio. La conferma del nostro parere possiamo trovarla nel fatto che ci incontriamo con quest'uso del trapassato remoto nei testi medievali latini che precedono la formazione delle lingue neolatine. ${ }^{13}$ Dunque, se questa forma esprimeva un preterito del passato a se aveva un significato enfatico nella principale prima del Mille, possiamo supporre che alla fine abbia perduto elementi stilistici.

2. ;e si assettò, pregando la donna che facesse presto. Questa in un tratto ebbe imbandito: e tosto cominciò a tempestare il suo viandante d'inchieste, (b, XVI, p. 279).

Questo tipo d'applicazione del trapassato remoto non si allontana dal precedente. Ma qui incontriamo un nuovo momento: l'espressività del trapassato remoto, cioè la rapidità dell'azione o, meglio, la sua perfettività non viene data solo con la forma verbale, ma si sottolinea per mezzo di «in un tratto», cioè di un avverbio o di una locuzione avverbiale.

Qui dobbiamo tornare a Pietro Bembo, il primo dei grammatici italiani che s'interessò di questo fenomeno, sebbene non possiamo essere d'accordo con il suo parere, volendo egli vedere in questa forma, pur nella principale, un tempo relativo, la cui espressività tenta di spiegare con certi avverbi con cui questa forma verbale sta di solito. ${ }^{14}$

Tornando all'ultima redazione del romanzo manzoniano, possiamo dire che in ambo i casi trovati c'incontriamo nelle indipendenti col tipo del trapassato remoto che ci spiega il Bembo, cioè si tratta dei tempi la cui perfettività è stata sottolineata da avverbi oppure da locuzioni

11 Quest'affermazione vale solo per le indipendenti e non per le dipendenti.

${ }_{12} \mathrm{Cfr}$. p. e., A. Kalepky, Ein fiktives Tempus in der romanischen Sprachmissenschaft (Archioum Romanicum, XIII, 1929, p. 548-556).

${ }^{13}$ Cfr. Ernst Gamillscheg, Studien zur Vorgeschichte einer romanischen Tempuslehre, Wien, 1913, p. 259-60.

14 Prose della volgar lingua, Torino, sine anno, libro III, p. 129-31. 
avverbiali (in un momento, subito): «Questa, in un momento, ebbe messo la tabola;》 (c, XVI, p. 279); «Ma il tono di quella voce, l'aspetto, il contegno, e soprattutto le parole di Federigo, l'ebbero subito rianimate,» (c, XXIV, p. 419).

$$
* * *
$$

Volendo dare una spiegazione linguistica a questo fenomeno, non possiamo limitarci esclusivamente alla lingua italiana. Dobbiamo ricordare che lo stesso fenomeno esiste nel francese. ${ }^{15}$ Secondo noi, si tratta di un fenomeno comune, strettamente legato a quello dello spostamento dei tempi, che ebbe luogo nel periodo precedente la formazione delle lingue romanze (p.e. AMAVISSET amasse). Ma più tardi, nel tempo storico della loro vita, le lingue romanze hanno avuto ciascuna una propria via. P. e., l'italiano continuò il processo (ricordiamo soltanto l'uso del condizionale passato invece del condizionale presente come futuro in relazione al passato), e il francese creò nel frattempo le forme pluricomposte (il a $\mathrm{bu}$, il a eu $\mathrm{vu}$ ).

Tornando adesso di nuovo al tempo in parola, dobbiamo dire che i grammatici francesi, discutendone l'applicazione nella principale, non vedono fra il passé antérieur e il passé simple una differenza più grande di quella che esiste fra l'uso del passé surcomposé e del passé composé. ${ }^{16}$ Senza dubbio, la stessa differenza fra i due tempi corrispondenti deve trovarsi anche nell'italiano. Però, in ogni modo, non si tratta di nessuna differenza temporale, ma solo d'aspetto.

Ripetiamo ancora una volta quello che volevamo metter in rilievo in questo lavoro: l'unico uso del trapassato remoto è di indicare un'azione finita, cioè questa forma verbale rappresenta un passato remoto un po' allargato; se lo troviamo oggi per lo più nelle temporali, ciò non deriva dalla sua natura temporale ma dalla sua posizione. La sua rarissima applicazione odierna non è d'accordo soltanto col lento ma sempre più evidente spegnersi del passato remoto (un processo già terminato nel Settentrione), ma è legato, direi, piuttosto coll'intenzione dei grammatici di vedere nel passato remoto un remoto. Quanto al Mezzogiorno, il cosiddeto passato remoto non è tale; è per lo più un aoristo, e perciò può segnare un'azione avveratasi nel momento temporale del narratore, p.e.: «Stamattina mi alzai alle cinque». In questa applicazione, sostituendo il passato remoto, il trapassato remoto continua a vivere nel Sud. ${ }^{17}$ Dunque, il trapassato remoto rappresenta solo un passato remoto sottolineato per rilevare meglio la fine dell'azione.

15 Maurice Grevisse, Le Bon Usage, sixième édition, Paris, 1955, p. 578-79.

16 Jacques Damourette et Édouard Pichon, Des mots à la pensée, Essai de grammaire de la langue française, Paris, 1936, tome V, p. 452-53 (§ 1853) e p. $297-300$ (\$ 1776).

17 Gerhard Rohlfs, Historische Grammatik der italienischen Sprache und ihrer Mundarten, Band II, p. 482-83, Bern, 1949. 
Questa affermazione sarà accettata se sappiamo che esiste soltanto l'attivo del trapassato remoto, mentre il suo passivo è introvabile (eccetto nei manuali grammaticali che tentano di dare un ordine esponendo la morfologia). ${ }^{18}$ Questo fenomeno però è facilmente spiegabile: se si tratta di un'azione esiste la possibilità dell'uso del trapassato remoto invece del passato remoto; quanto al passivo, trattandosi di uno stato, che come tale rappresenta anche dal punto di vista logico qualcosa di durativo, la sostituzione del trapassato remoto al passato remoto perde ogni senso. Perciò si trovano solo gli esempi in cui il trapassato remoto dell'attivo sta parallelamente col passato remoto del passivo, come: «Ma poiché fu venuta la fine del desinare, e le vivande furono rimosse, ancora più lieti che prima, cominciarono a cantare,» (Dec., VII, introd., p. 446-47).

\section{Rezime}

\section{TRAPASATO REMOTO U TRIMA REDAKCIJAMA MANCONIJEVOG ROMANA}

Zadržavajući se na trapasatu remotu, jednom vremenu koga je u italijanskom jeziku iz veka u vek sve manje, u trima redakcijama Manconijevog romana, autor beleži sve pojedinačne slučajeve $i$ izlaže ih tabelarno i procentualno prema vrsti rečenica u kojima se nalaze (nezavisnim ili pojedinim vrstama zavisnih). Konstatujući da se ovo vreme do danas održalo jedino u temporalnim. rečenicama, autor podvlači da je ono u ranijim vekovima bilo prilično često i u ostalim vrstama zavisnih rečenica kao i u nezavisnim (u poslednjima se povremeno sreće i u Manconijevom delu).

Sto se tiče njegove funkcije, autor je sklon da u njemu vidi apsolutno vreme, naglašavajući da mu funkciju relativnog vremena obično daju same temporalne rečenice u kojima se najčešće nalazi.

Naposletku, on u njemu vidi istu funkciju koju nosi i pasato remoto, samo nešto snažnije izraženu složenim oblikom. Svoje tvrdjenje potkrepljuje i time što konstatuje da ovo vreme ne može imati pasiva, pa navodi primere u kojima se na istom vremenskom stepenu nalazi aktiv trapasata remota i pasiv pasata remota.

${ }^{18}$ Cfr. M. D. Savić, op. cit., pagg. 30-33. 